\title{
Enhanced spectral brightness via coherent laser beam shaping (Conference Presentation) (Withdrawal Notice)
}

Michael Soskind, Pawel Kaczmarek, Krzysztof Abramski, Gerard Wysocki

Michael G. Soskind, Pawel Kaczmarek, Krzysztof Abramski, Gerard Wysocki, "Enhanced spectral brightness via coherent laser beam shaping (Conference Presentation) (Withdrawal Notice)," Proc. SPIE 11410, Laser Radar Technology and Applications XXV, 114100E (8 June 2020); doi: $10.1117 / 12.2558734$

\section{SPIE. Event: SPIE Defense + Commercial Sensing, 2020, Online Only}




\section{Enhanced spectral brightness via coherent laser beam shaping (Conference Presentation) (Withdrawal Notice)}

Michael G. Soskind, and Gerard Wysocki

Princeton Univ. (United States)

Pawel Kaczmarek, and Krzysztof Abramski

Wroclaw Univ. of Science and Technology (Poland)

Proceedings Volume 11410 Laser Radar Technology and Applications XXV; $114100 E$ (2020)

https://doi.org/10.1117/12.2506838

Event: SPIE Defense + Commerical Sensing, 2020, Online Only, United States

Online Publication Date: 27 April 2020

Withdrawn from Publication: 8 June 2020

Publisher's Note: This conference presentation, originally published on 27 April 2020, was withdrawn on 8 June 2020 per author request. 